\title{
Quaderni
}

QUADERNI Communication, technologies, pouvoir

\section{L'hôpital à l'épreuve de la performance économique : doctrines, instruments et hybridations des valeurs}

\section{Christelle Routelous}

\section{(2) OpenEdition}

\section{Journals}

\section{Édition électronique}

URL : http://journals.openedition.org/quaderni/735

DOI : $10.4000 /$ quaderni.735

ISSN : 2105-2956

Éditeur

Les éditions de la Maison des sciences de l'Homme

\section{Édition imprimée}

Date de publication : 5 octobre 2013

Pagination : 5-16

Référence électronique

Christelle Routelous, "L'hôpital à l'épreuve de la performance économique : doctrines, instruments et hybridations des valeurs », Quaderni [En ligne], 82 | Automne 2013, mis en ligne le 05 octobre 2015, consulté le 19 avril 2019. URL : http://journals.openedition.org/quaderni/735 ; DOI : 10.4000/ quaderni.735 


\section{$D$ ossier}

\section{L'hôpital à \\ l'épreuve de \\ la performance économique : doctrines, instruments et hybridations des valeurs}

\section{Christelle Routelous}

Docteure en sociologie EA Management des Organisations en Santé - Institut du Management,

École des Hautes Etudes en Santé Publique, Rennes-Paris.

\section{Managérialisme et État}

Depuis quelques années, les réformes dans le secteur public s'accélèrent et ce, en particulier, à l'hôpital. La volonté affichée est celle d'obtenir une meilleure efficience du secteur public et la maîtrise des dépenses.

\section{La réduction des dépenses publiques comme enjeu partagé}

Les dépenses de santé ont très fortement augmenté ces quarante dernières années. La croissance des dépenses de santé est un phénomène commun à la plupart des pays développés et est, en partie, liée à des phénomènes structurels tels que les progrès des techniques médicales, l'apparition de nouvelles pathologies, le vieillissement de la population. Les premières politiques mises en œuvre ont privilégié une approche quantitative des problèmes de santé. Elles ont principalement reposé sur la maîtrise de l'offre. Face aux limites des politiques quantitatives et sectorielles de mâ̂trise des dépenses de santé, les pouvoirs publics ont démultiplié les mesures de régulation du secteur hospitalier. Ces réformes semblent passer par l'importation et la transposition des modèles issus du nouveau management public (NMP).

Défini simplement, le NMP constitue un ensemble de doctrines, développé par accumulation, qui promeut de nouvelles manières de penser l'organisation administrative à partir d'un ensemble hétérogène de représentations tirées de théories économiques, de prescriptions issues de savoirs de management, de descriptions de pratiques expérimentées dans des réformes, en particulier dans les pays anglo-saxons et de systématisations 
produites par des organismes supranationaux ${ }^{1}$.

Ses préconisations, qui ont quelques peu varié ces vingt dernières années, mobilisent cinq principes d'organisation, déclinés en différentes mesures : la séparation entre les fonctions de stratégie, de pilotage et de contrôle et les fonctions opérationnelles de mise en œuvre et d'exécution; la fragmentation des bureaucraties verticales par création d'unités administratives autonomes (les agences), par décentralisation ou par empowerment de groupes d'usagers; le recours systématique aux mécanismes de marché (concurrence entre acteurs publics et avec le secteur privé, individualisation des incitations, externalisation de l'offre) ; la transformation de la structure hiérarchique de l'administration en renforçant les responsabilités et l'autonomie des échelons en charge de la mise en œuvre de l'action de l'État; la mise en place d'une gestion par les résultats fondés sur la réalisation d'objectifs, la mesure et l'évaluation des performances et de nouvelles formes de contrôle dans le cadre de programmes de contractualisation.

Ainsi, les idées néo-managériales ont été diffusées par l'État, à travers ces nouvelles réglementations qui valorisent les logiques de marché, l'efficience économique, la responsabilisation des établissements et des équipes soignantes, le contrôle de la performance.

\section{La transposition des modèles existants}

Des réformes similaires ont été observées dans de nombreux autres pays européens (Oomkens ${ }^{2}$, 2010, Kuhlmann et al ${ }^{3}$., 2009, Kirkpatrick ${ }^{4}$ et al., 2009). Dans ce numéro, P. Garel nous décrit comment la Commission Européenne, le Fonds Monétaire International et la Banque Centrale Européenne ont imposé des mesures très précises de changement dans les systèmes de santé, en contrepartie de soutiens financiers. L'auteur indique que tout en maintenant le principe de la compétence des États membres sur l'organisation et le financement des systèmes de santé, des instruments politiques et législatifs sont venus apporter de nombreuses exceptions à la capacité des États membres à garder l'intégralité de cette compétence à travers le développement du marché intérieur ou la coordination européenne des politiques macroéconomiques. L'attention portée à l'amélioration de la viabilité des finances publiques et, en particulier, celles concernant les soins de santé renforce l'influence de l'Union Européenne et des autorités supranationales sur la gouvernance du secteur de la santé.

Cette dynamique est relayée par les gouvernements à travers la mobilisation de modèles transposés d'autres pays ${ }^{5}$. Sous couvert de dépolitisation de la décision, la comparaison internationale devient un instrument d'action publique à part entière. Ainsi, comme le souligne S. Guigner, l'« evidence-based policy » élude à la fois les dimensions politiques, stratégiques et cognitives de l'action publique. La comparaison des politiques publiques dépolitise et rationalise l'action publique en alimentant un double apprentissage : l'apprentissage des gouvernants et celui des opposants.

Ainsi, sans même qu'ils aient prouvé leur efficacité, ce sont les mêmes outils influencés par le NMP qui sont importés par les pouvoirs publics français. Les principes de la nouvelle gouver- 
nance publique sous-entendent l'existence de notions, de fondements économiques universels, génériques applicables à tous les types d'organisation.

\section{Managérialisme et santé}

Mobilisant les instruments séparément ou en les combinant dans des programmes ambitieux, de nombreuses réformes ont été lancées, ces dernières années, dans le secteur de la santé.

Une volonté ancienne de maîtriser l'offre hospitalière

Alors que la France connaît une expansion économique, la loi du 31 décembre 1970 instaure une carte sanitaire. La France est ainsi divisée en secteurs sanitaires afin d'autoriser la construction d'un plateau technique minimal, avec radiologie, bloc opératoire et laboratoires d'analyses médicales. Cette loi se caractérise par la volonté de permettre une égalité d'accès aux soins. Mais la maîtrise des dépenses publiques joue un rôle très important dans les réformes qui ont suivi ${ }^{6}$. Le mouvement législatif marque la détermination des pouvoirs publics de recentrer les hôpitaux sur le plateau technique au détriment de la mission hôtelière. La loi du 31 juillet 1991 a pour objectifs de réduire le nombre de lits en s'appuyant sur une planification de plus en plus contraignante. Le schéma régional d'organisation des soins (SROS) est créé pour favoriser les restructurations hospitalières. L'autorité de régulation indique par ce biais les services qui sont amenés à fermer, se restructurer ou fusionner avec d'autres.

L'ordonnance du 24 avril 1996 relative à la réforme de l'hospitalisation publique et privée créée les Agences Régionales de l'Hospitalisation. Les objectifs clairement avoués par les pouvoirs publics étaient de réduire le parc hospitalier français de près du tiers de sa capacité, en lien avec la réduction importante des durées de séjours des malades hospitalisés, grâce à une plus grande concentration des équipements hospitaliers. Les moyens pour y parvenir sont la responsabilisation des acteurs, la contractualisation et la coordination entre établissements publics.

L'ordonnance $n^{\circ}$ 2005-406 du 2 mai 2005 simplifiant le régime juridique des établissements de santé instaure un nouveau mode de financement : la nouvelle tarification à l'activité (T2A), qui schématiquement privilégie les recettes sur les dépenses, les résultats sur les moyens. Le budget s'appelle désormais l'« état prévisionnel des recettes et des dépenses ».

La loi du 21 juillet 2009 portant réforme de l'hôpital et relative aux patients, à la santé et aux territoires a pour objectif de réorganiser et de moderniser l'ensemble du système de santé. Le texte prévoit la création de communautés hospitalières de territoire ou de partenariat publicprivé pour permettre la mise en commun des moyens de plusieurs établissements dans une logique de subsidiarité et de gradation des soins allant des structures de proximité aux plateaux techniques les plus sophistiqués.

En 2009, l'Agence Nationale d'Appui à la Performance des établissements de santé et médicosociaux est créée et a pour missions d'aider les établissements de santé et médico-sociaux à améliorer leur organisation et leur gestion en 
élaborant et en diffusant des recommandations et des outils. Elle en assure le suivi de la mise en œuvre, pour permettre aux établissements de moderniser leur gestion, d'optimiser leur patrimoine immobilier, de suivre et de maîtriser leur performance. Dans le cadre de son programme, elle peut procéder ou faire procéder à des audits de la gestion ou de l'organisation de l'ensemble des activités des établissements.

À travers ces différentes mesures ce sont le plafonnement des dépenses et des gains de productivité qui sont visés.

\section{Contractualisation, paiement à l'acte et res- ponsabilisation}

Les pouvoirs publics français ont importé des dispositifs devant favoriser la performance des hôpitaux en transposant trois principales mesures. La contractualisation avec les agences régionales, le changement de mode de financement et la responsabilisation des médecins dans les choix de gestion.

D'une part, on peut souligner la mise en place d'agences (agences régionales d'hospitalisation, agence régionale de la santé, agence nationale d'appuis à la performance) qui relaient les orientations nationales dans les régions. Ces agences ont un pouvoir de contrainte croissant sur les établissements de santé (notation des directeurs d'hôpital) et sur leurs stratégies (contractualisation sur les objectifs stratégiques).

D'autre part, la mise en place d'un paiement à l'acte se substituant à une enveloppe globale, apparaît comme le principal levier pour réaliser des gains de productivité. J.-C. Moisdon, décrit dans ce numéro, le nouveau mode de financement des établissements de santé français, pensé selon un principe fort d'incitatif à l'efficience. Comme la plupart des pays développés, la France met en place la tarification à l'activité, pour remplacer un système de financement par budget alloué, jugé inéquitable et surtout peu efficient. Ce nouveau mode de financement repose sur le développement d'un système d'information médico-économique devant permettre une transparence sur la nature des séjours hospitaliers et la consommation de soins. Ce dispositif remanié à de nombreuses reprises doit permettre d'estimer les coûts par séjour et par groupes homogènes de malades. Cet outil vise une connaissance fine $\mathrm{du}$ niveau de dépenses et d'activité. Les tarifs pour le secteur public sont différents de ceux pratiqués pour le secteur privé, même s'il a été question un temps de les faire converger.

La mise en place de la T2A s'est accompagnée à de la possibilité pour les établissements de santé de signer des « contrats de retour à l'équilibre financier » avec les agences régionales d'hospitalisation puis les agences régionales de santé créées en 2010. Les établissements s'y engagent à équilibrer leur budget, dans un cadre toutefois réglementé par les pouvoirs publics, qui fixent l'augmentation annuelle des dépenses de santé par le biais de l'ONDAM. Ils sont notamment incités à optimiser leurs coûts, maîtriser les durées de séjour, procéder à des prises en charge ambulatoires. C'est donc toute leur stratégie qui est touchée par cette réforme. La pression s'accroît chaque année car les tarifs sont revus à la baisse régulièrement, ce qui engage des établissements à accroître leur activité pour obtenir des ressources 
identiques à l'année précédente.

Enfin, on peut évoquer l'apprentissage partagé des enjeux de maîtrise des dépenses et de la responsabilisation face à l'emploi des ressources, par les directeurs et les médecins qui donnent lieu à des contractualisations en cascade. Le processus est récent, mais le cheminement a été construit en plusieurs étapes. D'abord, on a mis l'accent sur la nécessité d'inscrire l'organisation dans une logique de maîtrise des dépenses. L'état des comptes nationaux, le contexte de crise économique, le poids du système de santé dans les comptes nations, sont des discours omniprésents qui finissent par ne plus être questionnés. Ensuite, on a assisté à une critique partagée des dirigeants des établissements de santé et de la communauté médicale. En effet, ce sont tout autant les gestionnaires que des professionnels du soin qui sont critiqués pour leurs insuffisances en matière de gestion et d'organisations des soins. Enfin, l'accent est mis sur l'environnement complexe, sur les problématiques de déficit en ressources médicales, sur les coûts élevés et l'obsolescence rapide des plateaux techniques, sur la compétitivité et l'attractivité des cliniques...

L'hôpital se doit de devenir une organisation « performante » où l'ensemble des acteurs sont responsabilisés. Dans ce sens, se sont succédées ces dernières années de nombreuses réformes tentant d'inscrire médecins et directions dans les mêmes logiques gestionnaires. Depuis 2005, une cogestion médecin-administration est construite pour « mieux gérer» les hôpitaux. La gouvernance est exercée par un conseil exécutif qui, présidé par le directeur chef de l'établissement, sera composé à parité des responsables de l'administration désignés par le directeur et des médecins, présidents de la commission médicale d'établissement (CME), et responsables des pôles. En 2009, le Sénat a modifié le texte pour donner plus de poids aux médecins dans la gouvernance de l'hôpital : le président de la CME, médecin élu par ses pairs, coordonnera la politique médicale avec le directeur, et non plus sous son autorité, comme le stipulait le texte initial. Le président de la CME est vice-président du directoire. Il élabore avec le directeur et en conformité avec le contrat pluriannuel d'objectifs et de moyens, le projet médical d'établissement. L'organisation interne des établissements publics de santé est définie en pôles d'activité. Le directeur signe avec le médecin responsable de pôle un contrat précisant les objectifs et les moyens, après avis, du président de la CME pour vérifier la cohérence du contrat avec le projet médical. Le chef de pôle met en œuvre la politique du pôle, en cohérence avec la politique de l'établissement et organise, avec les équipes médicales, soignantes et administratives d'encadrement, sur lesquelles il a autorité fonctionnelle, le fonctionnement du pôle et l'affectation des ressources humaines. Ainsi, il s'agit de responsabiliser les médecins face aux contraintes économiques. L'organisation par pôles permet une visibilité en matière de dépenses et de recettes des services. Une place plus importante des décisions budgétaires est laissée aux médecins dans les choix réalisés en matière d'allocation de ressources.

La responsabilisation gestionnaire des établissements de santé et des médecins en particulier, les engage à négocier avec leur culture professionnelle et organisationnelle pour solutionner les problèmes relatifs à la maîtrise des dépenses. Les 
professionnels doivent se persuader du bien-fondé de ces finalités et des mesures à mettre en œuvre.

\section{Performance économique et hôpitaux publics}

La recherche de la performance économique, tout comme la nécessité de maîtriser les dépenses publiques, voire de les réduire, sont des principes qui ne font plus l'objet de débats et sont admis comme allant de soi. Reste que, dans les faits, cela apparaît de prime abord comme difficilement conciliable pour l'hôpital public.

\section{Missions du public, positionnement territorial et stratégies possibles}

La «performance hospitalière » pose le problème des stratégies possibles de l'hôpital public, garant des missions de service public. En effet, les établissements de santé doivent veiller à leur situation financière et donc modifier leur organisation tout en continuant à offrir des soins pour tous et sans dégrader leur qualité. L'analyse de la rentabilité économique doit prendre en compte des facteurs structurels de l'établissement (activités, facteurs de production, spécialisation) et des facteurs contextuels (concurrence, part de marché). En outre, elle devrait idéalement tenir compte de la qualité des soins délivrés?

Des travaux tentent d'identifier les facteurs explicatifs de l'efficience économique des établissements de santé. Selon les auteurs ${ }^{8}$, quatre catégories de variables influant sur la performance sont généralement mises en évidence : organisationnelles, managériales, case-mix et facteurs de marché. Les facteurs organisationnels font référence aux caractéristiques fondamentales de l'établissement (taille, statut, mission d'enseignement, etc.). Les variables managériales reflètent la stratégie d'allocation des ressources, d'optimisation des coûts et d'investissement (renouvellement des immobilisations, dettes, intensité du travail, etc.). Les variables de casemix décrivent l'activité de l'établissement du point de vue tant de l'éventail des pathologies traitées que des caractéristiques des patients. Et enfin, les facteurs de marché (concentration des hôpitaux, salaire moyen, densité de médecins, revenus des patients, etc.) reflètent à la fois les caractéristiques socio-économiques de l'environnement et la concurrence.

Les résultats mettent en évidence un effet gamme. Les établissements très spécialisés sont plus rentables que ceux ayant un large éventail de prise en charges. Au-delà, un taux d'occupation élevé et des durées de séjour plus courtes sont synonymes de plus grande performance économique. Or, ces stratégies ne sont pas toujours possibles pour les établissements publics. En effet, les hôpitaux publics, en tant qu'hôpitaux de proximité et du fait de leurs missions de service public, ne peuvent adopter des stratégies de niche comme le font les cliniques et ont du mal à réduire les durées de séjours pour les personnes âgées en perte d'autonomie ou très isolées socialement. Ainsi, même si l'efficience est devenue dans la période récente un enjeu majeur pour les établissements de santé en raison notamment de la tarification à l'activité, la réalisation de gains de productivité est à mettre en lien avec leurs contraintes et les publics pris en charge. Les contraintes pesant sur les établissements de santé sont très liées à leur statut, leur positionnement en termes d'activité offerte, le statut de leurs personnels ou des mis- 
sions de service public.

Ainsi, après avoir travaillé à réduire la masse salariale, une des stratégies possibles est la croissance de l'activité et de la productivité. Dans ce sens, sur la période 2003-2009, les résultats (Boisguérin et Minotier, 2012) mettent en évidence une hausse continue de la productivité en court séjour pour les établissements publics de santé, comme le confirme les travaux sur le suivi de la tarification à l'activité (Moisdon, 2013).

\section{L'accommodement et l'hybridation des logiques médicales}

Ainsi, il semblerait que l'incitatif économique conduise les établissements à réformer leurs processus de soins, pour accroître leur productivité. Dans ce cadre, on est en droit de s'interroger sur le positionnement des médecins, dont on connaît à la fois la capacité de résistance et l'autonomie décisionnaire'. Ce d'autant que l'on présente de manière traditionnelle, les rapports entre professions et nouvelle gestion publique dans une dimension conflictuelle (Bèzes, 2011). De même, on prend pour acquis que professionnels et gestionnaires sont profondément différents, aussi bien dans leur rapport à l'organisation dans laquelle ils évoluent, que du point de vue des valeurs qui les animent ${ }^{10}$.

Nous avons évoqué ci-dessus l'enrôlement des médecins dans les choix budgétaires dans le cadre de la contractualisation des pôles. Les médecins ne doivent alors plus seulement se préoccuper de la conformité de leur activité avec les bonnes pratiques mais aussi prendre en compte les moyens à mobiliser pour les réaliser. Les soignants sont confrontés à plusieurs défis : ils doivent offrir des soins de qualité à toutes les personnes qui en ont besoin tout en veillant à une mobilisation au plus juste de leurs ressources pour limiter les coûts. Ces défis sont à la fois organisationnels et économiques mais aussi éthiques. Le rôle de plus en plus prégnant des médecins dans la gestion des services, l'acceptation des systèmes d'informations et de contrôle de l'activité ne doit pas faire disparaitre les oppositions de valeurs sous-jacentes. Cette hybridation entre logique médicale et logique gestionnaire soulève un certain nombre de questions, comme l'illustre dans ce numéro, les travaux de Pierru et de Marche.

Nous verrons comme Derber et Schwartz ${ }^{11}$ (1991) l'indiquent, qu'il est possible de mettre en cohérence les buts des professionnels et ceux de l'organisation, l'autonomie des médecins et la nécessité de suivi des critères d'efficience et de comptabilité. Pour cela, il faut considérer l'acceptation de nouvelles responsabilités, non comme le signe d'un renoncement des médecins à leur autonomie ou d'une subordination aveugle aux principes managériaux, au contraire. Le concept de « responsabilisation de l'autonomie » $\left(\right.$ Dent $^{12}$, 2003) réduit la traditionnelle dichotomie entre professionnels et gestionnaires par l'intermédiaire de concepts communs tels que la compétitivité et la survie économique de l'établissement.

\section{Rationnaliser et rationner : quand les fins justifient les moyens}

La question que nous nous posons ici est quelles appropriations font les médecins de ces contraintes économiques et de rationalisation des prises en 
charges. Pour prolonger les travaux sur l'apprentissage des outils de gestion, il nous a semblé pertinent de saisir comment les valeurs véhiculées par les dernières réformes sont mobilisées dans les pratiques par la profession médicale et comment elles engendrent de nouvelles relations avec les patients, l'environnement et l'éthique professionnelle. Dans les services, la gestion des flux de patients et ses implications organisationnelles deviennent un enjeu prédominant. La $\mathrm{T} 2 \mathrm{~A}$ incite les services à démultiplier le nombre de prises en charge, ce qui implique de réduire la durée des séjours et l'accompagnement, alors que le système d'enveloppe fermée finançant les missions d'intérêt général, pousse les médecins à limiter le nombre de bénéficiaires ou d'actes.

Gérer la contrainte budgétaire dans des situations humainement douloureuses, c'est ce que nous propose $\mathrm{H}$. Marche dans le cas des patients atteints de cancer et pris en charge en soins palliatifs. Dans un contexte de fin de vie et face à la réduction de la durée des séjours, les soignants mettent en place des stratégies particulières pour gérer l'accueil, le maintien ou le transfert des malades. Ces négociations donnent lieu à des conceptions différentes du « sujet de soin ». Le travail relationnel évolue selon les incertitudes médicales, mais aussi beaucoup en fonction des contraintes organisationnelles. Les médecins réalisent un travail de responsabilisation des malades qui tend à s'accroître dans des situations de fortes pressions organisationnelles. Étonnamment, l'auteur souligne que c'est dans le comportement des malades et de leur entourage que les soignants fondent leurs insatisfactions vis-à-vis du travail relationnel et non dans les contraintes organisationnelles du service.
Autre exemple, celui des permanences d'accès aux soins de santé financées par des enveloppes spécifiques. Là aussi, le « rationnement » des prises en charge est aussi réalisé à la discrétion des professionnels de terrain. F. PIERRU décrit dans son article la multiplication des « choix pathétiques » que doivent opérer les soignants confrontés à l'afflux de population de migrants présentant des pathologies lourdes, aggravées par une grande précarité sociale, économique et juridique, dans un contexte de renforcement des contraintes budgétaires et de durcissement des politiques d'immigration. Ce travail analyse les manières de faire ordinaires et les arrangements informels quotidiens avec la réglementation. Reste que pour l'auteur, certains segments de la profession médicale, en raison notamment de la position occupée dans la division du travail et la hiérarchie médicale, sont particulièrement prédisposés à intégrer une partie des critiques gestionnaires et les nouvelles règles de gestion.

Confrontés à une enveloppe budgétaire limitative pour prendre en charge un afflux de patients lourds et précaires et les prescriptions coûteuses des spécialistes hospitaliers, les soignants n'ont pour seule issue que la possibilité de faire avec, tout en tentant de faire au mieux en construisant des « critères objectifs d'éligibilité ». Cette rationalisation des choix lève les foudres des associations de défenses des droits des malades/ migrants qui voient dans ce procédé de la discrimination et du rationnement des soins et non les contraintes imposées aux soignants.

Ainsi, on assiste à un réordonnancement des objectifs de prise en charge, qui fait la part belle aux enjeux financiers et qui est construit par un 
déploiement d'instruments de pilotage (contrat de pôle, tarification à l'activité, information médicoéconomique par patients et par services, etc.) qui semble conduire les acteurs à intérioriser les enjeux de performance économique. Il apparaît que ces instruments de pilotage de la performance économique conditionnent les jeux de régulation croisée auxquels les soignants peuvent recourir pour desserrer ou contourner la contrainte et pour répondre aux enjeux de personnalisation de la prise en charge.

La nouvelle configuration institutionnelle et les tensions qui caractérisent le déploiement de ces nouvelles logiques semblent offrir des espaces pour l'émergence de nouvelles formes de régulation des prises en charge où la profession médicale a fait sienne les contraintes économiques. Celles-ci semblent avoir un impact sur l'usage et la définition des missions de l'hôpital, le positionnement des médecins et des usagers.

En effet, il semblerait que les zones d'incertitudes laissées par le cadre de l'action publique et les directions d'établissement, pour gérer les tensions dans les orientations et les priorités des pôles, engendrent une hybridation des logiques de recherche d'efficience d'une part, et d'individualisation des réponses d'autre part. Ici, l'approche globale médico-bio-psycho-sociale des malades est mobilisée à des fins de rationalisation des choix. L'innovation consiste à créer de nouvelles règles (formelles ou informelles) pour atteindre l'objectif gestionnaire. Les acteurs créent de nouveaux outils de rationalisation des dépenses et des ressources, par conviction, intérêt et/ou obligation. Le principal levier à cet effet, est la responsabilisation des malades et de leur entourage, la sélection des cas, l'alternance avec le domicile...

Toutefois, les partenaires administratifs, politiques, associatifs et les usagers eux-mêmes disposent de ressources pour tenter de contrôler eux aussi, pour partie, la qualité de la prise en charge et de l'accompagnement. Reste qu'il faut alors traduire et concilier préoccupations et priorités et que cela ne se fait pas sans tensions. Il nous semble à ce stade que l'analyse des tensions engendrées par la recherche de la performance économique à l'hôpital et de leurs résolutions, constitue un moyen privilégié de rendre compte des changements en cours. Ce, même si les acteurs semblent, en l'état encore au milieu du gué. 
$\mathrm{N} \cdot \mathrm{O} \cdot \mathrm{T} \cdot \mathrm{E} \cdot \mathrm{S}$

1. Bezès, Ph. et al., « New Public Management et les professions dans l'État : au-delà des oppositions, quelles recompositions ? », Sociologie du travail, $n^{\circ}$ 53, 2011, pp. 293-348.

2. Oomkens, R.F., « Professionals under Supervision: Performance-based Contracting in Hospital Care and Home Care », IRSPM Conference, April, Berne, Switzerland, 2010.

3. Kuhlmann, E., Allsop, J., Saks, M., « Professional Governance and Public Control: A Comparison of Healthcare in the United Kingdom and Germany », Current Sociology 57 (4), 2009, pp. 511-528.

4. Kirkpatrick, I., Kragh Jespersen, P., Dent, M., Neogy, I., « Medicine and Management in a Comparative Perspective: the Cases of England and Denmark ", Sociology of Health and Illness 31 (5), 2009, pp. 642-658. 5. Djelic, M.-L., "L'arrivée du management en France : un retour historique sur les liens entre managérialisme et État », Politiques et management public, vol.22, n², Tome 1, 2004, pp. 1-17.

6. Renucci A., Cueille, S., « Responsabilisation des acteurs dans les hôpitaux publics français et management : analyse des dernières réformes juridiques ", Politiques et management public, vol. 18-2, 2000, pp. 43-68.

7. Même si de nombreux indicateurs de qualité ont été développés, il s'agit plus d'indicateurs de qualité de certains processus que d'indicateurs de qualité globale des soins. Ils ne peuvent donc pas vraiment être mobilisés pour mesurer la performance qualitative des établissements. Néanmoins, il ne semble pas que la qualité des soins se soit dégradée depuis la mise en place de la T2A. Les résultats de l'enquête nationale sur les événements indésirables graves à l'hôpital montrent une stabilité entre 2004 et 2009, alors même qu'une partie des experts s'attendait à une hausse de la fréquence des événements indésirables sur cette période, du fait de la modification de la structure d'âge des patients hospitalisés, de la complexité technique des actes et des prises en charge, et des modifications des conditions de travail liées notamment aux réorganisations (in Le panorama des établissements de santé,édition 2011, sous la coordination de A. Exertier et Ch. Minodier, DREES).

8. Boisguérin, B. Minodier Ch., (2012), Le panorama des établissements de santé, édition 2012, Collection Études et statistiques, DREES.

9. Freidson, E., (1984), La profession médicale, Paris, Payot.

10. Exworthy, M.,Halford, S., (1999), «Professionals and Managers un a changing public sector : conflit, compromise and collaboration ?" in Professionals and the New Managérialism in the public sector, open university press, Buckingham-Philadelphie.

11. Derbert, R., Schwartz, W.A., " New mandarins or new proletariat? Professional power at work », Research in sociology of organization, 71-96.

12. Dent, M., (2003), Managing doctors, and saving a hospital : irony, rhetorique and actors networks, Organization, février, vol. 10, $\mathrm{n}^{\circ} 1$. 


\title{
$R \cdot E ́ \cdot S \cdot U \cdot M \cdot E ́$
}

L'optimisation des administrations publiques est un phénomène bien connu. Les politiques européennes convergent ces dernières années vers les mêmes solutions de réduction budgétaire pour l'ensemble des secteurs. Pensée unique et outils de contrôle semblent être les principaux leviers de cette révolution culturelle en dépit des nombreuses limites que rencontre la mise en œuvre des outils. L'introduction de logiques et d'outils du « new public management » interroge la concurrence de nouvelles valeurs avec celles qui fondent le service public. Or, la responsabilisation des professionnels et gestionnaires concernant l'utilisation de leurs ressources vient bouleverser à la fois les valeurs associées à la place, au rôle de l'hôpital, de même que les pratiques et le cadre des prises en charge.

\begin{abstract}
Optimization government is a known phenomenon. European policies converge to the same recent budget reduction solutions for all sectors. Single thought and control tools seem to be the main drivers of this cultural revolution despite many limitations faced by the implementation tools. The introduction of logic and tools of the «new public management» competition asks new values with those based on public service. Empowering professionals and managers regarding the use of their resources disrupts both the values associated with the place, the role of the hospital and practice.
\end{abstract}


